DESY 06-149

hep-ph/0611055

November, 2006

\title{
Spontaneous Non-thermal Leptogenesis in High-scale Inflation Models
}

\author{
Motoi Endo ${ }^{1}$ Fuminobu Takahashi $^{1}$ and T. T. Yanagida ${ }^{2,3}$ \\ ${ }^{1}$ Deutsches Elektronen Synchrotron DESY, Notkestrasse 85, \\ 22607 Hamburg, Germany \\ ${ }^{2}$ Department of Physics, University of Tokyo, \\ Tokyo 113-0033, Japan \\ ${ }^{3}$ Research Center for the Early Universe, University of Tokyo, \\ Tokyo 113-0033, Japan
}

\begin{abstract}
We argue that a non-thermal leptogenesis occurs spontaneously, without direct couplings of the inflaton with right-handed neutrinos, in a wide class of high-scale inflation models such as the chaotic and hybrid inflation. It is only a finite vacuum expectation value of the inflaton, or more precisely, a linear term in the Kähler potential, that is a prerequisite for the spontaneous non-thermal leptogenesis. To exemplify how it works, we show that a chaotic inflation model in supergravity naturally produces a right amount of baryon asymmetry via the spontaneous nonthermal leptogenesis. We also discuss the gravitino production from the inflaton.
\end{abstract}




\section{Introduction}

The origin of the baryon asymmetry in the universe is one of the most important issues in the particle cosmology. Among many baryogeneses proposed so far, particularly interesting is the leptogenesis scenario [1], which may be divided into two classes, thermal and non-thermal ones, depending on the mechanism to generate the heavy right-handed neutrinos. The thermal leptogenesis is simple, and therefore attractive; it almost automatically takes place once the cosmic temperature rises so high that the right-handed neutrinos are thermalized. Since it requires relatively high reheating temperatures, it is consistent with the big bang nucleosynthesis (BBN) only for a limited range of the gravitino mass. The non-thermal leptogenesis, on the other hand, is capable of producing the baryon asymmetry at lower reheating temperatures, allowing a broader range of the gravitino mass. However, since the generation of the right-handed neutrinos relies on couplings of the inflaton, one may get the impression that it needs ad hoc model-dependent assumptions in comparison with the thermal leptogenesis.

Recently there has been much progress concerning the decay processes of scalar fields such as moduli [2, 3, 4, 5] and inflaton [6, 7, 8] in supergravity. In particular, Ref. [8] has pointed out that, if the inflaton has a linear term in the Kähler potential \#1, the inflaton couples to all the fields that appear in the superpotential with gravitational strength \#2. The discovery of such couplings has opened a way to naturally induce the reheating into the visible sector. In particular, it enables the non-thermal leptogenesis [9, 10] to occur more naturally, in the sense that one does not have to introduce any direct couplings of the inflaton with the right-handed neutrinos.

In this paper we argue that the non-thermal leptogenesis naturally occurs in a certain class of high-scale inflation models \#3, without introducing couplings of the inflaton with the right-handed neutrinos. The presence of the linear term in the Kähler potential, which may result from the inflaton's VEV, plays an essential role in our discussion. To illustrate how it works, we investigate a chaotic inflation model in supergravity and show that the

\footnotetext{
\#1 Note that the Kähler potential contains a linear term when the inflaton acquires a finite vacuum expectation value (VEV), even if the minimal Kähler potential is assumed from the beginning.

\#2 T.T.Y. thanks T. Watari for a useful discussion on the point.

\#3 The inflaton must be heavy enough since otherwise the reheating temperature is too low for the leptogenesis to work. See Eq. (10) below.
} 
spontaneous non-thermal leptogenesis actually takes place and generates a right amount of the baryon asymmetry. Although we consider mainly the chaotic inflation model \#4, the results derived in the following are rather generic, and can be applied to any high-scale inflation models.

The paper is organized as follows. In Sec. 2 we review the chaotic inflation model in supergravity. In Sec. 3, we will discuss the spontaneous non-thermal leptogenesis. The last section is devoted to conclusions. The case without a linear term will also be briefly discussed in Appendix.

\section{Chaotic Inflation Model in Supergravity}

To construct a successful inflation model in supergravity, which we assume throughout this paper, there is a well-known $\eta$-problem [12]. The problem becomes much severer when constructing a chaotic inflation model [13], since the exponential prefactor of the scalar potential practically forbids any scalar fields to take values beyond the Planck scale, while the inflaton must initially sit at the point far beyond the Planck scale in the chaotic inflation model. It was pointed out in Ref. [14] that the problem can be solved by postulating a shift symmetry on the inflaton and the chaotic inflation in supergravity can be realized in a rather simple set-up.

According to Ref. [14], we assume that the Kähler potential $K\left(\phi, \phi^{\dagger}\right)$ is invariant under the shift of $\phi$,

$$
\phi \rightarrow \phi+i A
$$

where $A$ is a dimension-one real parameter, and we adopt the Planck unit: $M_{P}=1$ unless stated otherwise. Thus, the Kähler potential is a function of $\phi+\phi^{\dagger} ; K\left(\phi, \phi^{\dagger}\right)=$ $K\left(\phi+\phi^{\dagger}\right)=c\left(\phi+\phi^{\dagger}\right)+\frac{1}{2}\left(\phi+\phi^{\dagger}\right)^{2}+\cdots$, where $c$ is a real constant and must be smaller than $O(1)$ for a successful inflation. We will identify the imaginary part of $\phi$ with the inflaton field $\varphi \equiv \sqrt{2} \operatorname{Im}[\phi]$. Moreover, we introduce a small breaking term of the shift

\footnotetext{
\#4 The recent WMAP three year results have shown that the scalar spectral index is quite likely to be smaller than unity: $n_{s}=0.951_{-0.019}^{+0.015}$ 11. Among inflation models consistent with the WMAP results, from the observational point of view, the chaotic inflation model is interesting, because the predicted $\mathrm{B}$-mode signal in CMB polarization is likely to be detected by future observations such as Planck, Clover, and CMBpol.
} 
symmetry in the superpotential in order for the inflaton $\varphi$ to have a potential:

$$
W(\phi, \psi)=m \phi \psi,
$$

where we have introduced a new chiral multiplet $\psi$, and $m \simeq 2 \times 10^{13} \mathrm{GeV}$ represents the breaking scale of the shift symmetry and determines the inflaton mass.

The scalar potential is given by

$$
\begin{gathered}
V(\eta, \varphi, \psi)=m^{2} e^{K}\left[|\psi|^{2}\left(1+2\left(\eta+\frac{c}{\sqrt{2}}\right) \eta+\left(\eta+\frac{c}{\sqrt{2}}\right)^{2}\left(\eta^{2}+\varphi^{2}\right)\right)\right. \\
\left.+\frac{1}{2}\left(\eta^{2}+\varphi^{2}\right)\left(1-|\psi|^{2}+|\psi|^{4}\right)\right]
\end{gathered}
$$

with

$$
K=\left(\eta+\frac{c}{\sqrt{2}}\right)^{2}-\frac{c^{2}}{2}+|\psi|^{2},
$$

where we have assumed the minimal Kähler potential for $\psi$, and defined $\eta \equiv \sqrt{2} \operatorname{Re}[\phi]$. Note that $\eta$ and $\psi$ cannot be larger than the Planck scale, due to the prefactor $e^{K}$. On the other hand, $\varphi$ can be larger than the Planck scale, since $\varphi$ does not appear in $K$. For $\varphi \gg 1, \eta$ acquires the mass comparable to the Hubble parameter and quickly settles down to the minimum, $\eta \simeq-c / \sqrt{2}$. Then the scalar potential during inflation is given by

$$
V(\eta, \varphi, \psi) \simeq \frac{1}{2} m^{2} \varphi^{2}+m^{2}|\psi|^{2} .
$$

For $\varphi \gg 1$ and $|\psi|<1$, the $\varphi$ field dominates the potential and the chaotic inflation takes place (for details see Ref. [14]).

When $\varphi \simeq \sqrt{2}$, the slow-roll condition breaks down and the inflaton $\varphi$ starts to oscillate. The potential minimum after inflation is located at $\varphi=\eta=0$ and $\psi=0$ in the SUSY limit. Once we take account of the SUSY breaking \#5, the minimum slightly changes, although the shift is so tiny that the following discussion is not affected. The other important effect is the mixing between $\phi$ and $\psi^{\dagger}$ induced by the SUSY breaking. As

\footnotetext{
\#5 For a broad range of the gravitino mass from $O(10) \mathrm{eV}$ to $O(100) \mathrm{TeV}$, the Hubble parameter at the reheating must be smaller than the gravitino mass. Otherwise, too many gravitinos would be produced by particle scatterings in thermal plasma [6] unless there is late-time entropy production [15]. Therefore one needs to take account of the SUSY breaking when considering the inflaton decay.
} 
discussed in Ref. [6], $\phi$ and $\psi^{\dagger}$ almost maximally mix with each other to form the mass eigenstates:

$$
\varphi_{ \pm} \equiv \frac{\phi \pm \psi^{\dagger}}{\sqrt{2}}
$$

Therefore we will consider the decay processes of $\varphi_{ \pm}$instead of $\varphi, \eta$ and $\psi$.

\section{Spontaneous Non-thermal Leptogenesis}

The decay processes on which we focus our attention are those induced by the presence of the linear term in the Kähler potential: $\delta K=c\left(\phi+\phi^{\dagger}\right)$. Such a linear term is expected

to exist with a coefficient of order unity, $c \lesssim O(1) \# 6$, since it is consistent with the shift symmetry (11). Although the inflaton may have direct couplings with matter fields in the Kähler potential and the superpotential, we assume that such couplings are suppressed.

The inflaton can decay into all the fields that appear in the superpotential through the linear term with gravitational strength [8], if it is kinematically allowed, and we take up the following two important processes above all. First let us consider the inflaton decay through the top Yukawa coupling:

$$
W=Y_{t} T Q H_{u}
$$

where $Y_{t}$ is the top Yukawa coupling, and $T, Q$, and $H_{u}$ are the chiral supermultiplets of the right-handed top quark and left-handed quark doublet of the third generation, and up-type Higgs, respectively. The partial decay rate of the inflaton through the top Yukawa coupling is 8

$$
\Gamma_{T} \simeq \frac{3}{128 \pi^{3}}\left|Y_{t}\right|^{2}\left(\frac{c}{\sqrt{2}}\right)^{2} \frac{m^{3}}{M_{P}^{2}},
$$

where we have taken account of the mixing mentioned in the end of the previous section. Due to the decay process via the top Yukawa coupling, the reheating temperature $T_{R}$ is bounded below. We define the reheating temperature as

$$
T_{R} \equiv\left(\frac{\pi^{2} g_{*}}{10}\right)^{-\frac{1}{4}} \sqrt{\Gamma_{\varphi} M_{P}}
$$

\footnotetext{
${ }^{\# 6} c \lesssim O(1)$ is required for a successful inflation [14].
} 
where $g_{*}$ counts the relativistic degrees of freedom, and $\Gamma_{\varphi}$ denotes the total decay rate of the inflaton. Using $\Gamma_{\varphi} \geq \Gamma_{T}$, we obtain

$$
T_{R} \gtrsim 2 \times 10^{8} \mathrm{GeV}|c|\left(\frac{m}{2 \times 10^{13} \mathrm{GeV}}\right)^{\frac{3}{2}},
$$

where we have substituted $g_{*}=228.75$ and $Y_{t} \simeq 0.6 \# 7$, and the inequality is saturated if $\Gamma_{\varphi} \simeq \Gamma_{T}$. It is quite striking that the inflaton can decay into the visible sector even without direct couplings in the Kähler potential or the superpotential.

The other important process we consider is the decay into the right-handed (s)neutrinos thorough large Majorana mass terms:

$$
W=\frac{M_{i}}{2} N_{i} N_{i}
$$

where $i=1,2,3$ is the family index. We consider the inflaton decay into the lightest right-handed (s)neutrino $N_{1}$ for simplicity, assuming that the decay into the heavier ones, $N_{2}$ and $N_{3}$, are kinematically forbidden. We drop the family index in the following. The partial decay rate of the inflaton into the right-handed (s)neutrinos is [cf. [8]]

$$
\Gamma_{N} \simeq \frac{1}{16 \pi}\left(\frac{c}{\sqrt{2}}\right)^{2} \frac{m M^{2}}{M_{P}^{2}} \sqrt{1-\frac{4 M^{2}}{m^{2}}}
$$

where we have taken account of both the decay into the right-handed neutrinos and that into the right-handed sneutrinos. Since $\Gamma_{N}$ is proportional to $M^{2}$, it is much smaller than $\Gamma_{T}$ for $M \ll m$, but it can be comparable to or even larger than $\Gamma_{T}$ for $M=O\left(10^{12}\right) \mathrm{GeV}$.

The lepton asymmetry can be produced by the decay of the right-handed (s)neutrinos, if $C P$ is violated in the neutrino Yukawa matrix [1]. The resultant lepton asymmetry is given by

$$
\frac{n_{L}}{s} \simeq \frac{3}{2} \epsilon B_{N} \frac{T_{R}}{m}
$$

where $B_{N} \equiv \Gamma_{N} / \Gamma_{\varphi}$ denotes the branching ratio of the inflaton decay into the (s)neutrinos. The asymmetry parameter $\epsilon$ is given by [1, 16]

$$
\epsilon \simeq 2.0 \times 10^{-10}\left(\frac{M}{10^{6} \mathrm{GeV}}\right)\left(\frac{m_{\nu_{3}}}{0.05 \mathrm{eV}}\right) \delta_{\mathrm{eff}}
$$

\footnotetext{
\#7 Here we have estimated the top Yukawa coupling at an energy scale of the inflaton mass $\simeq O\left(10^{13}\right) \mathrm{GeV}$.
} 


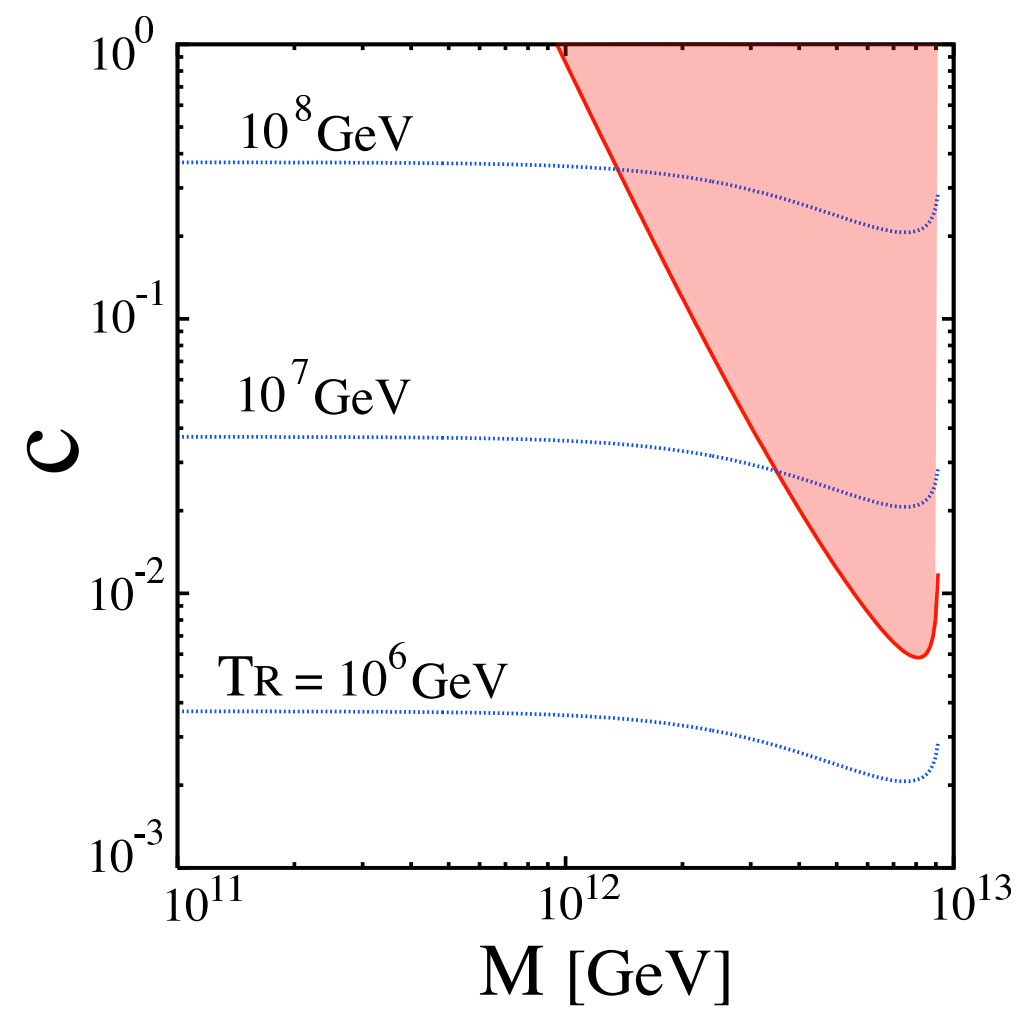

Figure 1: Contours of the reheating temperatures $T_{R}$, denoted by dotted (blue) lines. Taking account of the unknown $C P$ phase $\delta_{\text {eff }} \leq 1$, the shaded region above the solid (red) line can explain the present baryon asymmetry. We set $g_{*}=228.75, Y_{t}=0.6$ and $m_{\nu_{3}}=0.05 \mathrm{eV}$.

where $m_{\nu_{3}}$ is the heaviest neutrino mass and $\delta_{\text {eff }} \leq 1$ represents the effective $C P$-violating phase. The baryon asymmetry is obtained via the sphaleron effect: [17]

$$
\frac{n_{B}}{s}=-\frac{8}{23} \frac{n_{L}}{s} .
$$

Using the above relations, we obtain the right amount of baryon asymmetry,

$$
\frac{n_{B}}{s} \simeq 2 \times 10^{-10}|c|\left(\frac{M}{10^{12} \mathrm{GeV}}\right)^{3}\left(\frac{m}{2 \times 10^{13} \mathrm{GeV}}\right)^{-\frac{3}{2}}\left(\frac{m_{\nu_{3}}}{0.05 \mathrm{eV}}\right) \delta_{\mathrm{eff}},
$$

where we have approximated that the inequality (10) is saturated, assuming $M \ll m$.

In Fig. 1, we plot the contours of the reheating temperatures as a function of $c$ and $M$. Note that the total decay rate $\Gamma_{\varphi}$ is given by $\Gamma_{T}+\Gamma_{N}$, as long as $M<m / 2$. In addition, we show the region where the non-thermal leptogenesis can explain the baryon 
asymmetry measured by WMAP [11]:

$$
\left.\frac{n_{B}}{s}\right|_{\mathrm{WMAP}}=8.7_{-0.4}^{+0.3} \times 10^{-11} .
$$

From Fig. 1, one can see that the coefficient of the linear term in the Kähler potential, the reheating temperature and the (lightest) right-handed neutrino mass must be in the following ranges:

$$
\begin{aligned}
& 6 \times 10^{-3} \lesssim c \lesssim 1 \\
& 3 \times 10^{6} \mathrm{GeV} \lesssim T_{R} \lesssim 3 \times 10^{8} \mathrm{GeV} \text {, } \\
& 10^{12} \mathrm{GeV} \lesssim M \lesssim 10^{13} \mathrm{GeV}
\end{aligned}
$$

for the successful non-thermal leptogenesis. The parameter ranges shown above are the prediction of our non-thermal leptogenesis scenario in the chaotic inflation model. Note that such a large right-handed neutrino mass may induce large lepton flavor violating signals [18] at a detectable level by MEG [19].

The reheating temperature in the above range (19) constrains the gravitino mass to satisfy the bounds from the gravitino problem [20] \#8. The abundance of the gravitino produced by thermal scatterings is approximated by [23, 24]

$$
Y_{3 / 2} \simeq 1.9 \times 10^{-12}\left[1+\left(\frac{m_{\tilde{g}_{3}}^{2}}{3 m_{3 / 2}^{2}}\right)\right]\left(\frac{T_{\mathrm{R}}}{10^{10} \mathrm{GeV}}\right)
$$

where $m_{\tilde{g}_{3}}$ is the gluino mass evaluated at $T=T_{R}$, and we have dropped the logarithmic corrections for simplicity. If the gravitino is light, it may be the lightest SUSY particle (LSP) and therefore stable. The bounds on $T_{R}$ then come from the requirement that the gravitino abundance should not exceed the present dark matter (DM) abundance:

$$
T_{R} \lesssim \begin{cases}O(100) \mathrm{GeV} & \text { for } \quad m_{3 / 2} \simeq 10^{-2}-10^{2} \mathrm{keV} \\ 3 \times 10^{7} \mathrm{GeV}\left(\frac{m_{\tilde{g}_{3}}}{500 \mathrm{GeV}}\right)^{-2}\left(\frac{m_{3 / 2}}{1 \mathrm{GeV}}\right) & \text { for } \quad m_{3 / 2} \simeq 10^{-4}-10 \mathrm{GeV}\end{cases}
$$

On the other hand, if the gravitino is unstable, BBN puts severe constraints on $T_{R}$ :

$$
T_{R} \lesssim \begin{cases}(1-4) \times 10^{6} \mathrm{GeV} & \text { for } m_{3 / 2} \simeq 0.1-0.2 \mathrm{TeV} \\ 3 \times 10^{5}-4 \times 10^{6} \mathrm{GeV} & \text { for } m_{3 / 2} \simeq 0.2-2 \mathrm{TeV} \\ 5 \times 10^{5}-1 \times 10^{8} \mathrm{GeV} & \text { for } m_{3 / 2} \simeq 2-10 \mathrm{TeV} \\ (3-10) \times 10^{9} \mathrm{GeV} & \text { for } m_{3 / 2} \simeq 10-30 \mathrm{TeV}\end{cases}
$$

\footnotetext{
\#8 The recent results are given in Refs. 21] for the unstable gravitino and in Ref. 22] for the stable one. See Ref. [6] for the summarized results.
} 
for the hadronic branch $B_{h}=1$. For smaller $B_{h}$, the bounds are relaxed to some extent. For the heavy gravitino of mass $\gtrsim 30 \mathrm{TeV}$, no stringent constraints are obtained from BBN. However, another constraint comes from the abundance of the LSP produced by the gravitino decay:

$$
T_{R} \lesssim 9.3 \times 10^{9}\left(\frac{m_{3 / 2}}{100 \mathrm{TeV}}\right)^{-1} \mathrm{GeV}
$$

where we have assumed the anomaly-mediated SUSY breaking (AMSB) [25] with the wino LSP, and used the relation $m_{\tilde{W}} \simeq 2.7 \times 10^{-3} m_{3 / 2} \# 9$. Thus, the allowed range for $T_{R}$ (19) satisfies the bounds shown above, if the gravitino mass $m_{3 / 2}$ lies roughly in the following ranges: \#10

$$
\begin{aligned}
& \text { (i) } m_{3 / 2} \lesssim 10 \mathrm{eV} \\
& \text { (ii) } 10 \mathrm{MeV} \lesssim m_{3 / 2} \lesssim 10 \mathrm{GeV} \\
& \text { (iii) } m_{3 / 2} \gtrsim 10 \mathrm{TeV}
\end{aligned}
$$

For the case (i), the bound on $T_{R}$ does not exist, since the gravitino is so light that the contribution to the dark matter is negligibly small. For the cases (ii) and (iii), there are further constraints on the SUSY breaking sector from the non-thermal gravitino production by the inflaton decay [6], as will be discussed below.

Let us now discuss the gravitino pair production from the inflaton [6]. Even if the inflaton does not couple to the SUSY breaking field $z$, the gravitino pair production still occurs, and it is efficient especially if the SUSY breaking field $z$ has a large mass $m_{z}$ [3, 4] as in the dynamical SUSY breaking (DSB) scenario [29]. Assuming $m_{z}<m$ and no direct couplings between the inflaton and $z$, the gravitino production rate is [4]

$$
\Gamma_{3 / 2}^{(\text {pair })} \simeq \frac{|c|^{2}}{64 \pi} \frac{m^{3}}{M_{P}^{2}}\left(\frac{m_{z}}{m}\right)^{4} .
$$

The resultant gravitino abundance is

$$
Y_{3 / 2}^{(\text {pair })} \simeq 5 \times 10^{-4}|c|\left(\frac{m}{2 \times 10^{13} \mathrm{GeV}}\right)^{\frac{1}{2}}\left(\frac{m_{z}}{m}\right)^{4},
$$

\footnotetext{
\#9 This relation may be changed by radiative corrections [26].

\#10 Taking account of the decay of the next-to-lightest SUSY particle [27, 28, the upper bound of the case (ii) may become severer.
} 
where we have used (10) assuming $\Gamma_{\varphi} \simeq \Gamma_{T}$. Note that, since the gravitino abundance is proportional to the fourth power of the mass ratio $\left(m_{z} / m\right)$, it can be suppressed if $m_{z}$ is much smaller than the inflaton mass $m$. For instance, in the case of AMSB with the wino-like LSP, the bound on $Y_{3 / 2}$ is obtained from (21) and (24):

$$
Y_{3 / 2} \lesssim 2 \times 10^{-12}\left(\frac{100 \mathrm{TeV}}{m_{3 / 2}}\right)
$$

Using this bound, we obtain a mild constraint on $m_{z}$,

$$
m_{z} \lesssim 2 \times 10^{11} \mathrm{GeV}|c|^{-1 / 4}\left(\frac{m}{2 \times 10^{13} \mathrm{GeV}}\right)^{7 / 8}\left(\frac{m_{3 / 2}}{100 \mathrm{TeV}}\right)^{-1 / 4} \text { for AMSB. }
$$

On the other hand, for the lighter gravitino $(\lesssim 10 \mathrm{GeV})$ as in the gauge-mediated SUSY breaking (GMSB) [30] which corresponds to the case (ii), the gravitino should be the LSP. The bound on $Y_{3 / 2}$ reads

$$
Y_{3 / 2} \lesssim 5 \times 10^{-10}\left(\frac{1 \mathrm{GeV}}{m_{3 / 2}}\right) .
$$

Then the constraint on $m_{z}$ becomes

$$
m_{z} \lesssim 6 \times 10^{11} \mathrm{GeV}|c|^{-1 / 4}\left(\frac{m}{2 \times 10^{13} \mathrm{GeV}}\right)^{7 / 8}\left(\frac{m_{3 / 2}}{1 \mathrm{GeV}}\right)^{-1 / 4} \text { for GMSB }
$$

Since $m_{z}$ is likely smaller for the lighter gravitinos, the bound (31) is much milder than (29). Thus, although the gravitino pair production puts some constraints on the SUSY breaking models, they are not so severe \#11.

So far we have focused on the two decay processes among those induced by the linear term in the Kähler potential. As pointed out in Ref. [8], the gravitinos may be directly produced in a similar way, if the DSB sector has Yukawa couplings in the superpotential, and if the decay into the DSB sector is kinematically allowed. The gravitino production rate is expressed by

$$
\Gamma_{3 / 2}=\frac{\xi}{1536 \pi^{3}}\left(\frac{c}{\sqrt{2}}\right)^{2} \frac{m^{3}}{M_{P}^{2}},
$$

where $\xi$ is determined by the decay processes; the degrees of freedom of the decay products, the decay chains, the coupling constants of the Yukawa interactions in the SUSY breaking

\#11 Note that the gravity pair production severely constrains the gravity-mediated SUSY breaking models that contain a singlet with non-zero $F$-term, unless the inflaton has some unbroken symmetries in vacuum [6]. 
sector, and form factors of the hidden mesons and/or baryons. Although the constant $\xi$ strongly depends on the models, if all the Yukawa couplings are of order unity, $\xi$ is expected to be $O(1)$ or larger. The gravitino abundance is then

$$
Y_{3 / 2}=5 \times 10^{-7} \xi|c|\left(\frac{m}{2 \times 10^{13} \mathrm{GeV}}\right)^{\frac{1}{2}} .
$$

To avoid the gravitino overproduction, $\xi$ must be small enough. Using the upper bounds on $Y_{3 / 2}$, (28) and (30), we obtain

$$
\xi \lesssim \begin{cases}4 \times 10^{-6}|c|^{-1}\left(\frac{m}{2 \times 10^{13} \mathrm{GeV}}\right)^{-\frac{1}{2}}\left(\frac{m_{3 / 2}}{100 \mathrm{TeV}}\right)^{-1} & \text { for AMSB } \\ 1 \times 10^{-3}|c|^{-1}\left(\frac{m}{2 \times 10^{13} \mathrm{GeV}}\right)^{-\frac{1}{2}}\left(\frac{m_{3 / 2}}{1 \mathrm{GeV}}\right)^{-1} & \text { for GMSB }\end{cases}
$$

Thus, the Yukawa couplings of order unity in the DSB sector may not be allowed especially in the case of the AMSB scenario \#12. Note that there are the DSB models with no superpotential [31, 32, 33] (i.e., $\xi=0$ ), which evade the gravitino overproduction problem mentioned here \#13.

We have paid our attention so far to the baryon asymmetry generated via leptogenesis. For successful cosmology after inflation, a right amount of DM in addition to the baryon asymmetry must be generated. For the case (i) shown in (25), the gravitino should be the LSP, but it is too light to be the dominant component of DM. Therefore we need to introduce e.g. the axion to account for DM. In the case (ii), the gravitino can be DM, and it comes both from the thermal production and from the inflaton decay. In the case (iii), the LSP is likely the wino, which is also generated from the thermal production and the decay of the gravitinos.

\section{Discussion and Conclusions}

As we have seen in the previous section, the linear term in the Kähler potential plays an essential role in the spontaneous non-thermal leptogenesis scenario. Once the inflaton

\footnotetext{
\#12 It is marginally possible that the inflaton decay into the DSB sector is kinematically forbidden for $m_{3 / 2} \sim O(100) \mathrm{TeV}$. Then the constraint on $\xi$ is not applied.

\#13 The constraint on $\xi$ may be applicable even in this case, since the inflaton mass is close to the DSB scale in the AMSB scenario and there can be non-perturbative effects inducing the effective coupling $\xi_{\text {eff }}$ for composite states.
} 
acquires such a linear term, the non-thermal leptogenesis follows automatically. Although we have considered the chaotic inflation model to illustrate the mechanism, it can be applied to any high-scale inflation models in which the inflaton acquires a finite VEV, by replacing $c / \sqrt{2}$ with the VEV and $m$ with the inflaton mass of the model under consideration. Of course, the allowed ranges of the parameters and the constraints on the SUSY breaking sector from the gravitino overproduction problem depend on the inflation model.

For instance, in the case of the hybrid inflation model [34], the waterfall field obtains a finite $\operatorname{VEV}\langle\phi\rangle$, which is related to the inflaton mass $m_{\phi}$ as $m_{\phi}=\sqrt{2} \lambda\langle\phi\rangle$. Here $\lambda$ is a Yukawa coupling between the inflaton and the waterfall fields, and it takes a value ranging from $10^{-5}$ to $10^{-1}$ [35]. Let us concentrate on $\lambda \gtrsim 10^{-3}$, since otherwise the scalar spectral index would become close to unity, which is disfavored by the recent WMAP data [11. Then the VEV and the mass are given by $\langle\phi\rangle \simeq 2 \times 10^{-3}$ and $m_{\phi} \simeq 3 \times 10^{-3} \lambda$. Replacing $c / \sqrt{2}$ with $\langle\phi\rangle$ and $m$ with $m_{\phi}$ in Eq. (16), we obtain the baryon asymmetry produced by the spontaneous non-thermal leptogenesis in the hybrid inflation model:

$$
\left.\frac{n_{B}}{s}\right|_{\text {hybrid }} \simeq 9 \times 10^{-11}\left(\frac{\lambda}{10^{-3}}\right)^{3 / 2}\left(\frac{M}{m_{\phi} / 2}\right)^{3}\left(\frac{m_{\nu_{3}}}{0.05 \mathrm{eV}}\right) \delta_{\text {eff }} .
$$

Due to the smaller VEV $\langle\phi\rangle$ compared to $c$ in the chaotic inflation model, a right amount of the baryon asymmetry is generated only if $M$ takes a value close (but not too close) to the upper bound $m_{\phi} / 2$. Thus the spontaneous non-thermal leptogenesis works in the hybrid inflation model too. The constraints on the SUSY breaking sector can be similarly read from (29), (31) and (34) by replacing $c / \sqrt{2}$ with $\langle\phi\rangle$ and $m$ with $m_{\phi}$, and they are more or less similar.

In this paper we have investigated the reheating processes of the chaotic inflation in supergravity, paying particular attention to the decay processes induced by the linear term in the Kähler potential. We have found that a successful non-thermal leptogenesis takes place spontaneously, without direct couplings with the right-handed neutrinos. It requires the parameters such as $c, T_{R}, M$, and $m_{3 / 2}$ to be in certain ranges (see (18), (19), (20) and (25)). In particular, the gravitino mass either heavier or lighter than the weak scale is allowed, while the gravitino of a mass $O(1) \mathrm{TeV}$ encounters cosmological difficulties. Further, the gravitino production from the inflaton decay can be avoided if 
the SUSY breaking sector satisfies some constraints, which are not so severe. The chaotic inflation model we have investigated is therefore cosmologically viable, in the sense that it can naturally cause the leptogenesis avoiding the gravitino overproduction problem.

Lastly we should stress again that the spontaneous non-thermal leptogenesis scenario we have proposed in this paper can be applied to any high-scale inflation models if the inflaton has a finite VEV. The scenario is quite unique in that the non-thermal leptogenesis automatically occurs, and that it predicts several important parameters such as the reheating temperature, the right-handed neutrino mass and the gravitino mass, which may be probed in future experiments/observations.

\section{Acknowledgments}

The work of T.T.Y. has been supported in part by a Humboldt Research Award.

\section{A Case without a linear term}

Let us here briefly discuss the case without a linear term in Kähler potential. This can be realized by imposing a discrete symmetry on the inflaton potential. Since the linear term is suppressed by the discrete symmetry, the decay modes discussed in the text are ineffective. In particular, the non-thermal production of the gravitinos from the inflaton decay does not occur.

The reheating of the inflaton may occur through the couplings with (A) Higgs fields or (B) right-handed neutrinos. In the case $(\mathrm{A})$, we introduce the following coupling:

$$
W_{\text {int }}=h \phi H_{u} H_{d}
$$

where $h$ is a real numerical coefficient and is naturally small, $h=O\left(10^{-5}\right)$, since it breaks the shift symmetry (1). We show the charges of $U(1)_{R}$ and $Z_{2}$ symmetries on the inflaton

and the standard-model fields in Table 1 $\# 14$. The decay rate of the inflaton via the

\footnotetext{
\#14 The smallness of the $\mu$-term can be understood as a tiny breaking of the $Z_{2}$ symmetry.
} 
coupling Eq. (36) is

$$
\Gamma_{\varphi}^{(A)} \simeq \frac{1}{4 \pi}\left(\frac{h}{\sqrt{2}}\right)^{2} m
$$

The reheating temperature is given by

$$
T_{R} \simeq 3 \times 10^{9} \mathrm{GeV}\left(\frac{h}{10^{-5}}\right)\left(\frac{m}{2 \times 10^{13} \mathrm{GeV}}\right)^{\frac{1}{2}},
$$

which is high enough for thermal leptogenesis to work. In fact, for such high reheating temperatures, the right-handed neutrino with a mass $M \lesssim T_{R}$ reaches thermal equilibrium, and the baryon asymmetry is generated via thermal leptogenesis: [1, 16]

$$
\frac{n_{B}}{s} \simeq 3 \times 10^{-11}\left(\frac{\kappa}{0.1}\right)\left(\frac{M}{10^{9} \mathrm{GeV}}\right)\left(\frac{m_{\nu_{3}}}{0.05 \mathrm{eV}}\right) \delta_{\mathrm{eff}}
$$

where $\kappa$ denotes a suppression factor from the wash-out effect. In this case, the gravitino mass should be larger than $O(10) \mathrm{TeV}$ to satisfy the bounds from the gravitino problem (see $(23)$ ).

In the case (B) we consider the following interaction:

$$
W_{\mathrm{int}}=\frac{k}{2} \phi N N
$$

where $k$ is a real numerical coefficient of $O\left(10^{-5}\right)$ since it breaks the shift symmetry. The charge assignments are shown in Table 2. Here we promote $Z_{2}$ symmetry to $Z_{4}$ symmetry to accommodate the inflaton coupling with the right-handed neutrinos. The $Z_{4}$ symmetry may be identified with the subgroup of $U(1)_{B-L}$. The right-handed neutrino masses break the $Z_{4}$ symmetry down to $Z_{2}$ symmetry \#15. The decay rate is given by

$$
\Gamma_{\varphi}^{(B)} \simeq \frac{1}{16 \pi}\left(\frac{k}{\sqrt{2}}\right)^{2} m
$$

leading to the reheating temperature

$$
T_{R} \simeq 2 \times 10^{9} \mathrm{GeV}\left(\frac{k}{10^{-5}}\right)\left(\frac{m}{2 \times 10^{13} \mathrm{GeV}}\right)^{\frac{1}{2}} .
$$

\#15 The right-handed neutrino mass term induces the linear term of $\phi$ in the Kähler potential with a coefficient $c \sim O\left(10^{-2} M\right)$ at one-loop level. However, taking account of $M \lesssim 10^{15} \mathrm{GeV}$, the coefficient $c$ is so small that the inflaton decay through the induced linear term can be neglected. 


\begin{tabular}{|c|c|c|c|c|c|c|c|}
\hline \hline & $\phi$ & $\psi$ & $H_{u}$ & $H_{d}$ & $\mathbf{5}^{*}$ & $\mathbf{1 0}$ & $N$ \\
\hline$U(1)_{R}$ & 0 & 2 & $4 / 5$ & $6 / 5$ & $1 / 5$ & $3 / 5$ & 1 \\
\hline$Z_{2}$ & - & - & + & - & - & + & - \\
\hline \hline
\end{tabular}

Table 1: The charges of $U(1)_{R}$ and $Z_{2}$ symmetries in the case (A).

\begin{tabular}{|c|c|c|c|c|c|c|c|}
\hline \hline & $\phi$ & $\psi$ & $H_{u}$ & $H_{d}$ & $\mathbf{5}^{*}$ & $\mathbf{1 0}$ & $N$ \\
\hline$U(1)_{R}$ & 0 & 2 & 0 & 0 & 1 & 1 & 1 \\
\hline$Z_{4}$ & 2 & 2 & 2 & 2 & 1 & 1 & 1 \\
\hline \hline
\end{tabular}

Table 2: The charges of $U(1)_{R}$ and $Z_{4}$ symmetries in the case (B).

The non-thermal leptogenesis occurs in this case. Assuming that the reheating occurs mainly through the decay into the right-handed neutrinos, the baryon asymmetry is given by

$$
\frac{n_{B}}{s} \simeq 1 \times 10^{-10}\left(\frac{k}{10^{-5}}\right)\left(\frac{M}{10^{10} \mathrm{GeV}}\right)\left(\frac{m}{2 \times 10^{13} \mathrm{GeV}}\right)^{-\frac{1}{2}}\left(\frac{m_{\nu_{3}}}{0.05 \mathrm{eV}}\right) \delta_{\mathrm{eff}} .
$$

Note that a right amount of the baryon asymmetry is generated for $k \sim 10^{-8}$ and $M \sim$ $10^{13} \mathrm{GeV}$, corresponding to $T_{R} \sim 10^{6} \mathrm{GeV}$ being marginally compatible with the gravitino mass of $O(1) \mathrm{TeV}($ see (23) $)$ \#16.

Thus, if the inflaton has a discrete symmetry and the linear term in the Kähler potential is suppressed, either thermal or non-thermal leptogenesis is possible depending on the coupling with the matter fields. In particular, it should be noted that the dangerous decay processes such as the gravitino pair production and the decay into the SUSY breaking sector are suppressed by the discrete symmetry. Therefore there is no gravitino overproduction problem in this case.

\section{References}

[1] M. Fukugita and T. Yanagida, Phys. Lett. B 174, 45 (1986);

see, for a review, W. Buchmuller, R. D. Peccei and T. Yanagida, Ann. Rev. Nucl. Part. Sci. 55, 311 (2005).

\#16 However, there is the severe Polonyi problem [36] in this case, which cannot be solved in our framework. 
[2] M. Endo, K. Hamaguchi and F. Takahashi, Phys. Rev. Lett. 96, 211301 (2006);

S. Nakamura and M. Yamaguchi, Phys. Lett. B 638, 389 (2006).

[3] M. Dine, R. Kitano, A. Morisse and Y. Shirman, Phys. Rev. D 73, 123518 (2006).

[4] M. Endo, K. Hamaguchi and F. Takahashi, Phys. Rev. D 74, 023531 (2006).

[5] M. Endo and F. Takahashi, Phys. Rev. D 74, 063502 (2006)

[6] M. Kawasaki, F. Takahashi and T. T. Yanagida, Phys. Lett. B 638, 8 (2006); Phys. Rev. D 74, 043519 (2006).

[7] T. Asaka, S. Nakamura and M. Yamaguchi, Phys. Rev. D 74, 023520 (2006).

[8] M. Endo, M. Kawasaki, F. Takahashi and T. T. Yanagida, arXiv:hep-ph/0607170.

[9] T. Asaka, K. Hamaguchi, M. Kawasaki and T. Yanagida, Phys. Lett. B 464, 12 (1999); Phys. Rev. D 61, 083512 (2000);

G. F. Giudice, M. Peloso, A. Riotto and I. Tkachev, JHEP 9908, 014 (1999).

[10] See, for early works, G. Lazarides, C. Panagiotakopoulos and Q. Shafi, Phys. Lett. B 315, 325 (1993) [Erratum-ibid. B 317, 661 (1993)];

K. Kumekawa, T. Moroi and T. Yanagida, Prog. Theor. Phys. 92, 437 (1994).

[11] D. N. Spergel et al., arXiv:astro-ph/0603449.

[12] K. Kumekawa, T. Moroi and T. Yanagida, in [10];

E. J. Copeland, A. R. Liddle, D. H. Lyth, E. D. Stewart and D. Wands, Phys. Rev. D 49, 6410 (1994).

[13] A. D. Linde, Phys. Lett. B 129, 177 (1983).

[14] M. Kawasaki, M. Yamaguchi and T. Yanagida, Phys. Rev. Lett. 85, 3572 (2000); Phys. Rev. D 63, 103514 (2001).

[15] D. H. Lyth and E. D. Stewart, Phys. Rev. D 53, 1784 (1996);

M. Kawasaki and F. Takahashi, Phys. Lett. B 618, 1 (2005).

[16] L. Covi, E. Roulet and F. Vissani, Phys. Lett. B 384, 169 (1996);

M. Flanz, E. A. Paschos and U. Sarkar, Phys. Lett. B 345, 248 (1995) [Erratum-ibid. B 382, 447 (1996)];

W. Buchmuller and M. Plumacher, Int. J. Mod. Phys. A 15, 5047 (2000). 
[17] S. Y. Khlebnikov and M. E. Shaposhnikov, Nucl. Phys. B 308, 885 (1988);

J. A. Harvey and M. S. Turner, Phys. Rev. D 42, 3344 (1990).

[18] See, for example, K. Tobe, J. D. Wells and T. Yanagida, Phys. Rev. D 69, 035010 (2004).

[19] See http://meg.icepp.s.u-tokyo.ac.jp/ for the details of the MEG experiment.

[20] S. Weinberg, Phys. Rev. Lett. 48, 1303 (1982).

[21] K. Kohri, Phys. Rev. D 64, 043515 (2001); R. H. Cyburt, J. R. Ellis, B. D. Fields and K. A. Olive, Phys. Rev. D 67, 103521 (2003); K. Jedamzik, Phys. Rev. D 70, 063524 (2004); M. Kawasaki, K. Kohri and T. Moroi, Phys. Lett. B 625, 7 (2005); Phys. Rev. D 71, 083502 (2005); J. R. Ellis, K. A. Olive and E. Vangioni, Phys. Lett. B 619, 30 (2005); K. Kohri, T. Moroi and A. Yotsuyanagi, Phys. Rev. D 73, 123511 (2006).

[22] T. Moroi, H. Murayama and M. Yamaguchi, Phys. Lett. B 303, 289 (1993).

[23] M. Bolz, A. Brandenburg and W. Buchmuller, Nucl. Phys. B 606, 518 (2001);

see also J. Pradler and F. D. Steffen, arXiv:hep-ph/0608344.

[24] M. Kawasaki, K. Kohri and T. Moroi, in Refs. [21].

[25] L. Randall and R. Sundrum, Nucl. Phys. B 557, 79 (1999);

G. F. Giudice, M. A. Luty, H. Murayama and R. Rattazzi, JHEP 9812, 027 (1998);

J. A. Bagger, T. Moroi and E. Poppitz, JHEP 0004, 009 (2000).

[26] G. F. Giudice, M. A. Luty, H. Murayama and R. Rattazzi, JHEP 9812, 027 (1998);

T. Gherghetta, G. F. Giudice and J. D. Wells, Nucl. Phys. B 559, 27 (1999);

M. Ibe, T. Moroi and T. T. Yanagida, arXiv hep-ph/0610277.

[27] J. L. Feng, A. Rajaraman and F. Takayama, Phys. Rev. Lett. 91, 011302 (2003); Phys. Rev. D 68, 063504 (2003); J. L. Feng, S. Su and F. Takayama, Phys. Rev. D 70, 075019 (2004); J. R. Ellis, K. A. Olive, Y. Santoso and V. C. Spanos, Phys. Lett. B 588, 7 (2004); F. D. Steffen, JCAP 0609, 001 (2006); T. Kanzaki, M. Kawasaki, K. Kohri and T. Moroi, arXiv:hep-ph/0609246.

[28] M. Pospelov, arXiv:hep-ph/0605215; K. Kohri and F. Takayama, arXiv:hep-ph/0605243; M. Kaplinghat and A. Rajaraman, arXiv:astro-ph/0606209; 
R. H. Cyburt, J. Ellis, B. D. Fields, K. A. Olive and V. C. Spanos, arXiv:astro-ph/0608562.

[29] K. I. Izawa and T. Yanagida, Prog. Theor. Phys. 95, 829 (1996);

K. A. Intriligator and S. D. Thomas, Nucl. Phys. B 473, 121 (1996).

[30] M. Dine, A. E. Nelson and Y. Shirman, Phys. Rev. D 51 (1995) 1362; M. Dine, A. E. Nelson, Y. Nir and Y. Shirman, Phys. Rev. D 53 (1996) 2658; For a review, see, for example, G. F. Giudice and R. Rattazzi, Phys. Rep. 322 (1999) 419, and references therein.

[31] I. Affleck, M. Dine and N. Seiberg, Phys. Lett. B 137, 187 (1984); Phys. Lett. B 140, 59 (1984).

[32] I. Affleck, M. Dine and N. Seiberg, Phys. Rev. Lett. 52, 1677 (1984); Nucl. Phys. B 256, 557 (1985).

[33] H. Murayama, Phys. Lett. B 355, 187 (1995).

[34] E. J. Copeland, A. R. Liddle, D. H. Lyth, E. D. Stewart and D. Wands, in [12];

G. R. Dvali, Q. Shafi and R. K. Schaefer, Phys. Rev. Lett. 73, 1886 (1994);

A. D. Linde and A. Riotto, Phys. Rev. D 56, 1841 (1997).

[35] M. Bastero-Gil, S. F. King and Q. Shafi, arXiv:hep-ph/0604198.

[36] M. Ibe, Y. Shinbara and T. T. Yanagida, Phys. Lett. B 639, 534 (2006). 